\title{
La generalización de los derechos desde la perspectiva del
} feminismo de la igualdad

\section{The generalization of rights from a feminist perspective}

Yamid Enrique Cotrina Gulfo ${ }^{1 凶}$, Johana Victoria Ayala Redondo ${ }^{\circledR}$, Mateo Andres Obredor Jiménez $2 \bowtie$

1 Docente del Programa de Derecho de la Universidad del Atlántico, Barranquilla, Colombia. Máster en Estudios Avanzados en Derechos Humanos, Universidad Carlos III de Madrid. Máster en Derecho Constitucional.

2 Estudiante del Programa de Derecho de la Universidad del Atlántico, Barranquilla, Colombia.

Fecha correspondencia:

Recibido: enero 18 de 2021.

Revisado: agosto 20 de 2021.

Aceptado: septiembre 27 de 2021.

Forma de citar:

Cotrina, Yamid; Ayala, Johana

Victoria y Obredor, Mateo Andrés.

"La generalización de los derechos

desde la perspectiva del

feminismo de la igualdad" En:

Revista CES Derecho. Vol. 12, No.

2 , julio a diciembre de 2021,

p. 131-145.

https://dx.doi.org/10.21615/

cesder.6497

Open access

(D) Derecho de autor

Licencia creative commons

Ética de publicaciones

Revisión por pares

Gestión por Open Journal System

DOI: 10.21615/cesder.6497

ISSNe 2145-7719

Publica con nosotros

\section{Resumen}

La generalización de los derechos puede considerarse como un proceso inacabado, el cual genera efectos adversos a la universalidad e igualdad que plantea, y exponer la razón por la que necesita apoyo de procesos paralelos a este, tal es el caso de la especificación es fundamental para lograr la garantía de los derechos de las mujeres. Es por ello que se plantea la generalización como un proceso de ampliación hacia las mujeres como titulares de derechos y la especificación como proceso complementario en la profundización de su reconocimiento. Se debe partir de la positivación de los derechos, para plantear la necesidad de generalizar los derechos ya reconocidos desde su goce efectivo.

Palabras clave: derechos de las mujeres; necesidades; generalización; sufragio; feminismo.

\section{Abstract}

The generalization of rights can be considered an unfinished process, which generates adverse effects on the universality and equality that it proposes, and it is for this reason that it 
needs the support of parallel processes, such as the case of specification, which is fundamental to achieve the guarantee of women's rights. For this reason, generalization is proposed as a process of expansion towards women as holders of rights and specification as a complementary process in the deepening of their recognition. The starting point should be the positivization of rights, in order to propose the need to generalize the rights already recognized from their effective enjoyment.

Keywords: women's rights; needs; generalization; suffrage; feminism.

\section{Introducción}

El proceso de generalización hace parte de las cuatro líneas evolutivas de los derechos en las que figuran la positivación, la especificación y la internacionalización. De una forma secuencial, la generalización sería el proceso siguiente al de positivación -el cual hizo ingresar valores morales en el sistema jurídico- para que este segundo hiciera una función de expandir la titularidad de los mismos a más personas; así que este proceso con bastante influencia iusnaturalista, se puede definir como la ampliación de titularidad de los derechos a otros sectores de la sociedad que antes no poseían, dado por la necesidad de que todos realmente pudiesen ser reconocidos como titulares de estos y gozarlos efectivamente.

Este proceso nace como una necesidad por el reconocimiento de garantías y libertades fundamentales para el sostenimiento de los individuos; el objetivo de este es la extensión de la titularidad de los derechos a más sujetos de los que previamente se les otorgaba. Para ejemplificar, puede tomarse de referencia el derecho al sufragio que fue concedido a las mujeres, donde la necesidad de una igualdad tanto formal como material se hizo presente en la lucha para conseguir la posibilidad de votar bajo criterio propio, pero ¿realmente la generalización de este derecho fue eficiente para todas las mujeres?

La eficiencia de un derecho no está relacionado con el mero reconocimiento del mismo, y es por ello que hay diferentes puntos de la generalización que resultan cuestionables en el sentido de que su extensión de titularidad no comprende ciertas problemáticas, tal cual como comprender el concepto de 'universalidad de los derechos' desde un punto sesgado por el privilegio para pretender suplir los retos que diferentes comunidades enfrentan a la hora de ejercer sus derechos; es por ello que el análisis de los contextos de las diferentes comunidades marginadas es la tarea óptima para el desarrollo de políticas que busquen eliminar estos limitantes. 
Por lo tanto, la generalización de los derechos puede resultar siendo un proceso inacabado, debido a las diferentes barreras que suponen mujeres y diversas comunidades por el reconocimiento de sus derechos en diferentes partes del mundo, y por sí solo insuficiente debido a que el solo reconocimiento de los derechos no son el significado de su garantía, necesitando del proceso subsiguiente a él: la especificación, como apoyo para profundizar en los derechos que este expande, compartiendo una temporalidad paralela, y a su vez efectos simultáneos. Es decir, mientras el proceso de generalización puede ser representado como una expansión de los titulares de los derechos, en el caso de la especificación es visto como proceso de profundización, enfocándose en el goce efectivo de los derechos ya reconocidos a sus titulares.

\section{Metodología}

Esta investigación consta de ser una crítica realizada a la generalización de los derechos en la línea evolutiva, argumentando la razón por la cual este se considera un proceso inacabado en relación a los derechos de las mujeres y con aspectos cuestionables que generan efectos contrarios a la universalidad e igualdad que plantea, mediante casos concretos como la historia de discriminación que han sufrido las mujeres, demostrando también que necesita apoyo de procesos paralelos a él, como el de especificación, para que sus resultados sean más efectivos. Se tomará de manera concreta los textos de diferentes autores internacionales expertos en el tema como fuente de información, con el fin de indagar y conocer hechos, teorías y opiniones que permitan analizar el tema de estudio para consecuentemente realizar una visión crítica sobre el proceso de generalización de los derechos de las mujeres.

\section{Antecedentes}

En la etapa de positivación de la línea evolutiva de los derechos fue necesario juridificar los valores morales dados por el iusnaturalismo, integrando así los derechos en el sistema normativo. A partir de esto, se hace necesario que esos derechos sean debidamente extendidos, por lo que se procede a la generalización. Este proceso se puede evidenciar a través de la historia en las distintas declaraciones, como la francesa o la norteamericana.

La corriente iusnaturalista influyó de una manera bastante directa dentro de la generalización de los derechos -cuando esta afirma que los derechos deben ser para todos los hombres-, pues declaraciones como la de Independencia de los Estados Unidos de 1776, sostenía la igualdad de los hombres frente a otros; o en la Declaración de los derechos del hombre y del ciudadano en 1789 , de una distribución justa de derechos para todos. También en la Declaración de los derechos del Buen Pueblo de Virginia (EE.UU.) donde manifiesta que todos los hombres en su 
naturaleza son libres e independientes y que estos llegan al mundo con predeterminados derechos (Peces-Barba, 1987, p.18). Entonces, la generalización es un proceso donde los valores morales toman un lugar importante del mundo jurídico y donde la igualdad desarrolla ideas de bienestar común para los seres humanos.

Es preciso destacar que unos sectores de la sociedad presentaron obstáculos para ejercer varios derechos y que esa igualdad parecía condicionada a otros términos diferentes. Un ejemplo se evidencia en la historia de Estados Unidos, una llena de exclusiones, puesto que segregación, racismo y desigualdad -pues no "todos los hombres eran libres"- son las realidades que se vivieron y que aún quedan vestigios de esta.

Estas declaraciones mencionadas han sido el precedente clave para una generalización de los derechos en distintas partes del mundo, y que así mismo como los países referenciados antes donde se expidieron, presentaron problemas en la implementación de ciertos derechos y su extensión, los que fueron influenciados no serían ajenos a estos. A pesar de que se vea que los antecedentes del proceso datan de hace más de dos o tres siglos, la generalización sigue siendo un proceso el cual sigue en desarrollo.

\section{Las necesidades como fundamento de los derechos}

El debate que supone abordar el concepto "derechos fundamentales", supone también que detrás de ese derecho encontramos una necesidad fundamental. Estas necesidades básicas son aquellas de las que hablaba Maslow al referirse a ellas como "radicales" y así mismo, cumple su función en paralelo con los derechos civiles y políticos. Este tipo de necesidades son defectuosas, pues siempre mantiene una falla que es responsabilidad del Estado satisfacerlas. (Grassetti, 2018).

Los derechos civiles y políticos a la vez que tienen una relación con las necesidades básicas, estos poseen una función como dignificador de la vida humana (Zabala, Argüelles, 2018). Ese objetivo de los derechos es una característica importante la cual todo ser humano debe poseer; al presumir si alguien no tiene derecho al voto -el cual pertenece a los derechos civiles y políticos-, ¿qué papel puede jugar dentro la sociedad actual? Ninguno, en definitiva, esa persona no tiene voz en el sistema público, puesto que no participaría en el ejercicio de la democracia, no podría ser elegido ni elegir. 
Las necesidades han significado la carencia en la satisfacción de derechos dentro de una sociedad donde estas dan un impulso para fundamentar la prestación que se tiene para con el Estado por parte del sujeto. De manera más concreta, el objeto de la cuestión se centra en la garantía del derecho en cuestión; de igual forma, estas pueden considerarse fundamentadoras de derechos, pero no solo está presente la necesidad de que exista el derecho, sino también está la de que se pueda dar un goce efectivo de estos para todos, y por eso mismo es que esto abre paso a la necesidad de una generalización.

\section{La necesidad de una generalización de los derechos}

Tiempo antes de que comenzara este proceso se presumía que los derechos eran de todos por el simple hecho de existir, pero la diferencia de lo que se decía con lo que se hacía era abismal, como lo menciona Peces-Barba (1987):

Frente a la teórica afirmación de origen iusnaturalista de que los derechos eran para todos los hombres (...), la realidad era muy distinta. Algunos de los derechos no se extendían a todos, como la participación política o el sufragio, y se justificaba esta discriminación (pág., 28)

Por la causa anterior fue que se hizo necesario garantizar y expandir derechos para todos sin importar condiciones. Debía haber alguna forma en la que esos derechos que se decía que eran de todos, de verdad fuesen de todos y no de los que gozaban de las caras de los privilegios. De esta necesidad nace lo que se conoce como el proceso de generalización.

De acuerdo a Rafael de Asís (2006) la generalización sería “la extensión de la satisfacción de los derechos a sujetos y colectivos que no los poseían". El concepto de generalización es, entonces, la universalidad de un derecho que antes de esto estaba restringido a una clase económica o también llamada como 'burguesía'. En definitiva, la generalización trata de hacer una unificación entre una igualdad formal -un trato igualitario a todas las personas en la ley- y el de la universalidad (De Asís, 2006). Este proceso es importante porque hace posible que los derechos no queden retenidos en una clase social específica, sino que supone el goce efectivo de estos a más personas a través de la expansión de la titularidad esta vez para sujetos indeterminados, tal como se evidencia, por ejemplo, con el derecho al sufragio.

\section{El feminismo como aliado en la generalización del sufragio}

Lo que se conoce hoy en día como el derecho universal al sufragio es sin duda un gran ejemplo para mostrar el proceso de generalización de derechos, ya que la oportunidad de votar se vio 
permitida solo para ciertos sectores favorecidos durante gran parte de la historia, siendo un privilegio de los hombres burgueses y adinerados. Por lo tanto, el proceso del sufragio no se dio de manera absoluta, sino que tuvo que pasar por diferentes etapas para el reconocimiento de dicho derecho.

Históricamente, mientras el hombre sí podía ejercer su derecho al voto, la mujer era considerada un ser incapaz de pensar, irracional por naturaleza, por lo tanto, no tenía una vida jurídica. Incluso después de la revolución francesa se excluyó a la mujer y al colectivo femenino de atribuirle el reconocimiento merecido por su lucha en la libertad del pueblo francés, esta frase lo resume: "el feminismo es un hijo no querido de la llustración" (Valcárcel, 2001, p.8). Misoginia, machismo y una fuerte represión ha sido el pan de cada día para las mujeres.

Sin embargo, uno de los que se abrió en el campo de la lucha de igualdad de derechos para ambos sexos fue Poullain de la Barre en el siglo XVII, un seguidor de Descartes. Algunos se refieren a él como uno de los precursores del feminismo: "El primer feminista, la primera obra que podemos considerar sin matices que pertenece a la tradición política a la que podemos llamar feminismo es De la igualdad de los dos sexos" (Valcárcel, 2019). En esta obra, como dice su nombre, de la Barre argumenta que hay una igualdad entre ambos sexos y que la exclusión de la mujer se debió a una tradición mal entendida. (Valcárcel, 2019).

Por otro lado, Olimpia de Gouges, una pensadora del siglo XVIII, fue una de las que, en su valentía y rabia por la exclusión de la mujer de la esfera pública después de la revolución, feminizó la obra Declaración de los derechos del hombre y del ciudadano de forma que las palabras masculinas y todos sus derechos, pasaron a ser femeninas, atribuyendo los mismos derechos del hombre, a la mujer de la época; el trabajo de la autora marca un desarrollo progresista en su época en el epílogo de la obra en el que hace la descripción de la exclusión sufrida por la mujer después de la revolución (De Gouges, 1791). Este hecho, hizo que fuese llevada a la guillotina en 1793 (Álvarez, 2018).

Mary Wollstonecraft, una escritora inglesa que también se enfrentó con la realidad de su época, influenció bastante en la creación del posterior movimiento del sufragismo:

“(...) Con los sucesos revolucionarios franceses como punto de referencia, Inglaterra vería a la escritora Mary Wollstonecraft realizar un durísimo alegato contra los prejuicios de la época hacia la mujer, reclamando para ella la condición de ser racional, ser humano igual 
al hombre y, en consecuencia, acreedora de los mismos derechos y posibilidades de desarrollo personal" (Álvarez, 2018, p. 134)

Las anteriores autoras, dieron al proceso de generalización una base teórica para que las mujeres reclamaran su derecho al voto de forma universal. La formación de la teoría feminista da hincapié a la creación de asociaciones sufragistas en pro de este importante derecho, tal como lo fue la National Society for Woman's Suffrage (NSWS), una organización que a través de proyectos de ley pelearían por su derecho al voto (Álvarez, 2018), y alzarían su voz para que les respondan 'con hechos, no palabras', como lo hacían saber en su lema.

Este movimiento que es formado a mitad del siglo XIX, a través de las protestas, huelgas y demás formas de manifestaciones -incluso algunas bastante violentas-, las sufragistas lograrían su cometido en Inglaterra, en el cual las mujeres vienen a obtener su derecho en 1917, pero con 5 años más que los hombres requeridos para su ejercicio. Posteriormente, en 1928, se logra la igualdad en años entre hombres y mujeres necesarios para votar, generalizando así el sufragio. Este mismo proceso de la entrega del voto a la mujer fue dado en países como Estados Unidos -adelantándose a Inglaterra varios años más a través de la Decimonovena enmienda en 1920- y posteriormente en Francia -en 1944 a través del Comité français de la Libération nationale-. Teniendo en cuenta lo anterior, el proceso del reconocimiento del derecho al sufragio se realizó de manera progresiva para la búsqueda de la universalidad del derecho, es decir que la extensión de la titularidad del derecho al voto, ya sea para las mujeres y diferentes grupos a los que se les negó, pasó por una serie de etapas, para que el primer paso se diera en vías de la garantía del derecho: la lucha por la igualdad ante la ley.

Como se puede notar, el proceso de generalización se enfrentó a problemas, Pissarello (2009) menciona sobre este asunto:

(...) Recordar que la expansión de derechos, lejos de ser un fenómeno evolutivo o lineal, presupone necesariamente la idea de conflicto. Y es que la garantía de un derecho suele venir a menudo acompañada de la limitación o de la eliminación de privilegios (pág., 1).

De acuerdo a lo anterior, se entiende que para poder generalizar el sufragio fue necesario que clases invisibilizadas se alzaran en revolución y pelearan por lo que debía ser un derecho de todos, y no un privilegio de pocos. 


\section{Aspectos cuestionables del proceso de generalización}

\section{La universalidad situada en un punto de privilegio}

La generalización se relaciona con la universalidad al buscar la extensión de la titularidad de los derechos, pues la idea de lo universal implica una cobertura hacia todas las personas, pero no asegura que los resultados que se den sean igualmente generales, es decir, que la generalización se caracterice por la universalidad de los titulares de derecho, no significa que garantice el goce efectivo de estos. De hecho, esa misma titularidad puede dar como consecuencia directa a una universalidad que resultaría peligrosa. "Cuando se habla de una perspectiva "universal", en realidad se hace desde los valores del hombre (varón) blanco, occidental, adulto, etc. Es decir que lo universal tiene un punto de referencia muy particular para pretenderse como tal» (Jelin, 2012)

Esto demuestra que hay un sesgo dentro de esas políticas que apuntan hacia la "universalidad", pues se hacen desde un lugar de privilegio. De esta manera, habrá una falta de imparcialidad que no entenderá el contexto histórico y social de algunos sectores poblacionales determinados (Young, 2004). En definitiva, lo peligroso de esta perspectiva universal está en el hecho que no tendrá en cuenta la experiencia de ciertos sectores, sino lo vivido desde el lugar privilegiado de las personas a las que temas como el racismo, misoginia, transfobia, etc. no les afecta. Aquí es preciso, comenzar a hablar sobre la teoría de la interseccionalidad debido al análisis que se puede obtener después de tener en cuenta a factores como el sexo, el género, clase y raza. En esta teoría de la cual Kimberlé Crenshaw es precursora, se visibilizan las diferentes correlaciones entre una forma de opresión y otra, en las cuales se marginaliza a personas o comunidades por tener características que socialmente no son aceptada como lo explica el siguiente fragmento: "Expuso y trató de desmantelar las formas de marginación que operaban dentro de los discursos institucionalizados que legitimaban las relaciones de poder existentes" (Carbado, D., Crenshaw, K., Mays, V., \& Tomlinson, B., 2013, pág. 304).

La interseccionalidad da un análisis que explicaría a grandes rasgos problemas estructurales de la desigualdad social de acuerdo a cada caso individual (Vigoya, 2016, pág. 6). Teniendo en cuenta este análisis el cual abarca la mayor parte de las diferentes caras de la opresión, estaría descartando la posibilidad de soluciones que vengan desde un punto de vista que no conozca el contexto y realidad social de muchas personas que por razones de género, sexo, raza o clase son marginadas, imposibilitando el goce efectivo de sus derechos más fundamentales. Sino que el objetivo de esto es partir de esa diferencia y comprender los contextos de cada comunidad, con el fin de eliminar aquellas situaciones que signifiquen barreras para ejercer sus derechos. 
Es por ello que se afirma que: "Después de la violación (terrible caricatura del amor de la cual está ausente el consentimiento) la opresión es el segundo horror de la existencia humana. La opresión es una terrible caricatura de la obediencia" (Young, 2000). La opresión se puede generar a través de "cuestiones distributivas y aspectos importantes como procedimientos de toma de decisiones, división del trabajo y cultura" (Artigas, 2018, pág. 276). Es por lo tanto la consecuencia de la división y categorización de la sociedad que proviene de la misma división del trabajo, donde a partir de ahí, aquellas personas que no cumplían con los requisitos aceptados por la civilización en cada época, se marginaban.

Históricamente, la mujer se ha marginado de igual manera, pero no a todas de la misma forma, algunas mujeres eran aún más marginadas por ser negras o por su orientación sexual. La opresión no es igual para todas las personas. En definitiva, se puede decir que la generalización del sufragio no ha servido a todas las mujeres, puesto que, aunque ya tuviesen la oportunidad de votar y el reconocimiento, las situaciones de opresión a las que diferentes mujeres se han visto sometidas limitaban el ejercicio de sus derechos.

Hay una necesidad, por lo tanto, de que los derechos no tengan como una referencia centralista al hombre de occidente, y que la imparcialidad de estos derechos presente un punto más cercano a la realidad que entienda los retos a los que se puede enfrentar en su ejercicio los diferentes titulares de derechos. No basta con solo ejercerlo, sino que haya un ambiente óptimo y adecuado para su efectivo goce.

\section{El concepto de igualdad en la generalización}

El proceso de generalización ha intentado manejar una igualdad formal, pero esto no supone el hecho de que se goce de una igualdad verdadera, por el contrario, se puede notar irregularidades, como lo menciona Ruíz-Gálvez (2016):

“(...) Hasta el momento, el proceso de generalización no ha supuesto que las exclusiones se hayan eliminado totalmente, en la actualidad subsisten bajo formas renovadas en las democracias desarrolladas. Los trabajadores, las mujeres, los afroamericanos (en el caso específico de Estados Unidos) han alcanzado la plena igualdad jurídica, sin que ello signifique que se haya superado completamente su situación de discriminación y/o explotación (pág., 57)". 
Lo dicho deja entender que las desigualdades pueden subsistir en la actualidad bajo una aparente generalidad en los derechos; es un problema que se arraiga más al lado de una falta de efectividad dentro de las políticas que toma el Estado frente a este problema, que la generalización por sí sola no lo puede lidiar.

Si se observa el caso concreto de las mujeres se ve que la igualdad que propone la generalización no se ve efectuada, puesto que desde los privilegios siempre se ha hecho de lado al colectivo femenino, sometiéndose a una subordinación del que se puede afirmar que muchos de los derechos que se han conseguido generalizar para ellas ha sido por mera táctica para que los de la cima de la pirámide social sigan con el poder. De esto habla Iriarte Rivas (2018):

“(...) Aunque la mujer sea ciudadana, su ciudadanía es deficitaria; ya que mantiene, por una parte, su posición subordinada dentro de la jerarquía social, y, por otra, su subjetividad y realidad social no están presentes en el modelo de los derechos, ni en la noción de sujeto. Lo propio de su realidad material y condición de persona no es concordante con las condiciones para el pleno ejercicio de la ciudadanía y de los derechos (pág., 61)".

Lo anterior se puede evidenciar en el proceso de generalización del sufragio del que se ha hablado, ya que el derecho al voto femenino puede considerarse una estrategia de una parte de los privilegiados para mantener su estatus, porque, aunque las mujeres pudiesen sufragar, eso no implicaba que ellas tuviesen la autonomía para elegir, debido a la relación de subordinación con respecto de los varones en el que estas se encontraban, que, a la final, eran quienes decidían por ellas. Entonces, la generalización del voto femenino no sería por igualdad, sino por conveniencia de los de la cima de la pirámide social, por lo que se entiende que no fue un derecho concedido pensando en ellas.

Otro aspecto cuestionable en la generalización es que hablar de esta puede significar ignorar la diversidad. Al tratar de lograr una universalidad se está buscando la igualdad, pero no se tiene en cuenta que cada ser humano tiene necesidades, identidades y situaciones distintas, por lo tanto, la búsqueda de una igualdad es imposible porque todos son diferentes, pero la perspectiva de los beneficiados siempre es la que se va a aceptar y reconocer como correcta, y todo aquel que no encaje con las caras del privilegio van a encontrarse en desventaja, de modo que la igualdad es medible entonces con respecto a la relación que se tiene con los otros, se es desigual en relación a sus semejantes (Cotrina, 2020), y este es un punto en el que el proceso de generalización falla porque, como lo menciona Jelin (2012), todas las reivindicaciones 
planteadas en términos de derechos llevan a un paradigma de la igualdad pero, al mismo tiempo, las diferencias existen y es necesario reclamar el respeto de estas, por lo que se hace difícil mantener aquel ideal de igualdad universal propio del proceso.

\section{La generalización, un proceso en construcción}

Aunque aparentemente se haya conseguido una generalización de derechos, y se crea que fue algo de hace siglos, la realidad es otra. Este es un proceso que aún no termina y que siempre va a estar en constante cambio, tal como lo menciona Rey (2011):

"Igual que el de positivación, el proceso de generalización sigue abierto porque, por un lado, siguen existiendo sectores excluidos del reconocimiento de derechos (por ejemplo, las mujeres o determinadas minorías en muchos países del mundo) y, por otro, surgen nuevas realidades o desafíos a los derechos (pág., 118)".

En el caso de la mujer, en algunas partes del mundo generalizar sus derechos más básicos, puede significar un reto verdaderamente grande. La sociedad hermética de ahora, por razones de cultura o costumbres hace que este proceso se encuentre con problemas difíciles de resolver y que, debido a esta falta de garantías, las más perjudicadas de esto sean ellas.

Es por ello que la equidad de género es clave para combatir una desigualdad estructural en materia de los derechos de las mujeres:

"La equidad de género significa tomar conciencia y transformar la posición de desigualdad y subordinación que las mujeres han vivenciado a través de siglos de historia en relación a los hombres en la esfera familiar, económica, social, política, cultural y en la misma historia" (Duarte \& García, 2016, pág., 141).

En una sociedad cerrada a los valores como la libertad e igualdad, la generalización de los derechos de las mujeres - no solo en un sector occidental, sino en varias partes del mundo- no se dará de manera efectiva y la opresión, la exclusión y subordinación del sexo femenino no se detendrá.

Adicionalmente, dentro de esta construcción se postula una integración del proceso de especificación el que se supone el reconocimiento de derechos a sujetos y colectivos concretos que se encuentran en situaciones especiales, implicando por tanto una idea de igualdad 
material, como lo menciona De Asís (2006). La igualdad material que se traduce como "igualdad ante la ley" lo que se resume en un trato igualitario de las personas.

Para concluir, se hace necesaria la especificación como acompañante de la generalización ya que no solo son procesos que se dan paralelamente, sino que se complementan entre sí. En definitiva, la relación que estos dos procesos tienen es muy significativa porque la especificación entraría a integrar a la generalización, tratando de garantizar a los menos favorecidos los derechos que la última solo se preocupa por universalizar. La línea que los diferencia es muy delgada, porque de expandir derechos a todos, a garantizar el goce efectivo de estos para todos de determinado sector, solo hay un paso. La especificación no podría existir sin una generalización, pero sin la primera, la segunda estaría aún más incompleta.

\section{Conclusiones}

La satisfacción de necesidades puede ser considerada como la fundamentación de derechos, y así mismo es necesario que todos puedan gozar de ellos. Por eso es que surge el proceso de generalización, por la necesidad de expandir la titularidad de los derechos dados en el proceso de positivación. En esa misma línea se encuentra la especificación, que ayudará a garantizar el goce efectivo de estos.

La generalización de los derechos no puede tener una vista sesgada de las comunidades a quienes busca su reconocimiento y titularidad de derechos, pues comprender las situaciones y limitaciones de ese modo solo generará una solución precaria para los retos que busca solucionar. El análisis interseccional podrá dar una visión más general de aquellas barreras por las que pueden pasar las personas, partiendo de la desigualdad, y a partir de ahí dar una efectiva solución que garantice sus derechos y libertades.

Desde una perspectiva propia, el proceso de generalización y especificación son paralelos en temporalidad, y a su vez gozan de una perpendicularidad, ya que la primera cumpliría un efecto horizontal al tratar de expandir la titularidad de los derechos, pero esa universalidad genera ciertos vacíos cuestionables que desembocan en desigualdades, puesto que solo cumple con extender, no garantizar. Es ahí donde entra el efecto vertical de la especificación, ya que cumple el papel de profundizar en aquellos vacíos para tratar de garantizar el goce efectivo de los derechos a grupos de personas desfavorecidas, y es justo en ese punto donde se encuentran, tal como las líneas perpendiculares. 
Por otro lado, el hecho de que el proceso de generalización esté en armonía con la universalidad de los derechos no significa que estos hayan llegado a todos los seres humanos ni mucho menos que se haya acabado la desigualdad; por el contrario, hay lugares del mundo y minorías que aún luchan por ser titulares de estos. Sin embargo, es un proceso que sigue en construcción y constante cambio. Con el transcurso del tiempo la positivación avanza y la generalización debe seguirla para extender sus resultados, y ese precisamente es tal vez uno de los retos más grandes que tiene que enfrentar este proceso, tratar de efectuar la universalización con la existencia de tantos derechos y sectores a los que no puede llegar por barreras como la opresión de los Estados y los que miran desde las caras de los privilegios.

\section{Referencias}

Álvarez Rodríguez, I. (2018). El sufragio femenino en la II República. Revista de Derecho de La UNED (RDUNED), (22), 131. https://doi.org/10.5944/rduned.22.2018.22282

Artigas, A. (2018) "Opresión e interseccionalidad”, en Delgado, J. Vulnerabilidad, justicia y salud global (pp. 275-2849. Revista Internacional de Éticas Aplicadas. https://www.dilemata.net/revista/index.php/dilemata/article/view/412000196/563

Cotrina, Y. (2020). Necesidades como criterio fundamentador de los derechos humanos. UNIVERSITAS. Revista de Filosofía, Derecho y Política, (33), 85-100. https://doi.org/10.20318/universitas.2020.5520

Carbado, D., Crenshaw, K., Mays, V., \& Tomlinson, B. (2013). INTERSECTIONALITY: Mapping the Movements of a Theory. Du Bois Review: Social Science Research on Race, 10(2), 303312. doi:10.1017/S1742058X13000349

De Asís, R. (2006). Hacia una nueva generalización de los derechos. Un intento de hacer coherente la teoría de los derechos. En: CAMPOY, I. Una discusión sobre la universalidad de los derechos humanos y la inmigración, pp, 35-54 http://hd.handle.net/10016/9266

De Asís, R. (2018). Nuevas dimensiones del discurso de los derechos humanos. Studia Histórica. Historia Contemporánea, 36, 117-130. https://doi.org/10.14201/shhc201836117130

De Gouges, Olympe (1791). Declaración de los derechos de la mujer y de la ciudadana [consultado 11 Sep 2021]. Disponible en: http://www.culturamas.es/blog/2012/09/07/declaracion-de-los-derechos-de-la-mujery-de-la-ciudadana1791-por-olympe-de-gouges/ 
Duarte Cruz, J., García-Horta, J. (2016). Igualdad, Equidad de Género y Feminismo, una mirada histórica a la conquista de los derechos de las mujeres. Revista CS, (18), 107-158. https://doi.org/10.18046/recs.i18.1960

Grassetti, E. (2018). Satisfacción de necesidades y Modelo Social. Revista de Investigación en Psicología Social PSOCIAL, 4 (1), 6-29.

Iriarte, C. (2018). La discriminación estructural de género y su recepción sistémica en el sistema de derechos humanos. Anuario de Derechos Humanos, (14), 55-76. https://doi:10.5354/0718-2279.2018.49168

Jelin, E. (2012). Los derechos como resultado de luchas históricas. En Por los derechos. Mujeres y hombres en la acción colectiva (pp. 21-33) Nueva Trilce. https://doi.org/10.14409/extension.v1i2.454

Mackinnon, C. A. (1987). Feminism unmodified: Discourses on life and law. Harvard university press.

https://books.google.es/books?hl=es\&lr=\&id=rxE8FQzipYMC\&oi=fnd\&pg=PA1\&dq=C+ A.+Mackinnon\&ots=wivF4deZ60\&sig=XTMbtgg5s0xkk1M4p9RZCVxQWo\#v=onepage \&q=C\%20A.\%20Mackinnon\&f=false

Peces-Barba, G. (1987). Derechos fundamentales. Junta de Comunidades de Castilla-La Mancha. http://hdl.handle.net/10016/10462

Pisarello, G. (2009). Los derechos sociales y sus enemigos: elementos para una reconstrucción garantista. Defender y Repensar Los Derechos Sociales En Tiempos de Crisis., 13-23.

Rey Pérez, J. (2011). El discurso de los derechos: una introducción a los derechos humanos. Universidad Pontificia Comillas. https://books.google.es/books?hl=es\&lr=\&id=rbq3bsyOywwC\&oi=fnd\&pg=PA6\&dq=ge neralizaci\%C3\%B3n+derechos+incompleta\&ots=g2L2QNkewQ\&sig=kFRteRyXQ5MCFP7 JoEJydekxVpU\#v=onepage \&q\&f=false

Fernández Ruiz-Gálvez, M. E. (2016). La universalización efectiva de los derechos. Una tarea inacabada. Derecho \& Sociedad, (47), 53-63. Recuperado a partir de http://revistas.pucp.edu.pe/index.php/derechoysociedad/article/view/18873

Valcárcel, A. (2001). La memoria colectiva y los retos del feminismo. Unidad Mujer y Desarrollo, 1-60. Retrieved from http://socinfo.eclac.org/publicaciones/xml/7/13907//cl2022e.pdf 
Valcárcel, A. (2019). Ahora, feminismo. Cuestiones candentes y frentes abiertos. Madrid, MD: Cátedra.

Vigoya, M. V. (2016). La interseccionalidad: una aproximación situada a la dominación. Debate feminista, 52, 1-17.

Young, I. M. (2000). La justicia y la política de la diferencia (Vol. 59). Universitat de València. https://books.google.es/books?hl=es\&lr=\&id=8saLZGyel6sC\&oi=fnd\&pg=PA9\&dq=iris+ young+la+justicia $+\&$ ots $=$ cjdAfED $812 \&$ sig $=c 2 D d t y \times 2 E 4 X 4 \mathrm{mOq} 9 \mathrm{yDVb} 5 \mathrm{ZgE}-$ uY\#v=onepage \&q=iris\%20young\%20la\%20justicia\&f=false

Zabala, J. J. A., Argüelles, J. J. I. (2018). Derechos Humanos y Dignidad Humana. Iustitia Socialis: Revista Arbitrada de Ciencias Jurídicas y Criminalísticas, 3(4), 8-23. 